Verfügbar unter / Available at:

https://hdl.handle.net/20.500.11970/100284

Vorgeschlagene Zitierweise / Suggested citation:

Mohsin, Siddique; Tomita, Saki; Tajima, Yoshimitsu (2010): Experimental Study on Interaction of Waves, Currents and Dynamic Morphology Changes. In: Burns, Susan E.; Bhatia, Shobha K.; Avila, Catherine M. C.; Hunt, Beatrice E. (Hg.): Proceedings 5th International Conference on Scour and Erosion (ICSE-5), November 7-10, 2010, San Francisco, USA. Reston, Va.: American Society of Civil Engineers. S. 697-706. 


\title{
Experimental Study on Interaction of Waves, Currents and Dynamic Morphology Changes
}

\author{
Siddique Mohsin ${ }^{1}$, Saki Tomita ${ }^{2}$ and Yoshimitsu Tajima ${ }^{3}$ \\ ${ }^{1}$ Coastal Engineering Laboratory, Department of Civil Engineering., The University \\ of Tokyo, 7-3-1 Hongo Bunkyo-ku, Tokyo 113-8656, Japan; \\ email: voumohsin@coastal.t.u-tokyo.ac.jp \\ 2ditto; email: tomitasaki@coastal.t.u-tokyo.ac.jp \\ ${ }^{3}$ ditto; email: yoshitaji@coastal.t.u-tokyo.ac.jp
}

\begin{abstract}
Time-to-time interactive features among waves, currents and dynamic morphology changes are essential to quantitatively determine the beach erosion problems. This study puts special focus on the impact of dynamically changing beach profiles on the wave and current properties. We first developed a new set of imagecapturing techniques that enabled us to obtain high-resolution quantitative data sets of waves, currents and bottom bed profiles both in time and space domains. In a $2 \mathrm{D}$ wave flume, multiple high-speed cameras and normal video cameras were installed beside the flume to capture the time-varying bottom bed profiles and spatial distributions of instantaneous current velocity and surface water fluctuations across the entire surf zone. Brightness of the obtained image was transferred to the instantaneous suspended sediment concentrations. The obtained data sets successfully describe the formation and onshore movement of the bars until they reach to the equilibrium state.
\end{abstract}

\section{INRTODUCTION}

As the wave shoals on the sloping beach in the surf zone, its profile dramatically changes. Initially, waves slow down in the surf zone and starts gaining height as they propagate on sloping beach until they reach to a breaking depth where waves are forced to break. At this height, wave crest deforms to forward-leaning profile and yields surface roller that collapses in front of the wave crest. This complicated nonlinear phenomenon of wave breaking generates turbulence which plays vital role in transferring wave energy, momentum, and mass fluxes into the water body in the surf zone (Huang et al., 2009). The generated turbulence reaches to the bottom and picks up bottom sediments in suspension. Suspended sediments are then transported by instantaneous and mean current velocity and cause morphology changes which, in turn, affect the nearshore hydrodynamics. This interactive process continues until the beach reaches to a state of equilibrium.

To predict the beach erosion and topography changes along beach with a greater accuracy, detailed knowledge of the flow parameters such as spatial-temporal surface water elevations, the corresponding internal flow dynamics and particularly their interactions with morphology are crucial. Other important parameters, such as time averaged currents, turbulence and sediment transport can readily be estimated based on obtained spatial instantaneous current velocities. 
Our understandings of near-surf-physics are usually based on quantitative measurements carried out during several laboratory studies. However, most of these experimental studies are limited to wave breakings on uniformly sloping fixed bed beaches. Traditionally, two types of techniques have often been used to investigate changes in water surface profile of waves and its underwater dynamics. The first approach is to apply point measurement techniques such as Acoustic Doppler Velocimetry, (ADV) or Laser Doppler Velocimetery, (LDV) in combination with wave gauges ( e.g., Elgar et al., 2005, Stive, 1980, Nadaoka et al., 1989, Ting and Kirby, 1996, Cox and Kobayashi, 2000, Shin and Cox, 2006 and Longo, 2009). Over equilibrium profile of natural sandy beaches, Hurther, et al. (2007) used ADV and optical back scatters (OBS) to study the turbulent energy production and dissipation under broken irregular waves. The second approach is full-field measurements by the use of the state of art Particle Image Velocimetry (PIV) technique. Recently, studies by Govender et. al., (2002), Govender et. al. (2004), Kimmoun and Branger (2007) demonstrated the skills of full-field PIV measurements over sloping fixed bed beaches in wave flumes. The results are overwhelming and present details about the fluctuating velocity components and various features of turbulence kinetic energy such as dissipation, production, advection, convection during various wave phases. In order to capture the interactive features of changing bed profiles and surrounding wave and current fields, it is essential to obtain synchronized high-resolution data sets of both current and bottom bed profiles.

Therefore, the present study aims to develop a new set of image-capturing techniques that enable us to obtain synchronized high-resolution quantitative data sets of waves, currents and movable bottom bed profiles both in the time and space domains across the entire surf zone. High speed video cameras (HSVCs) and normal video cameras are utilized to reveal the instantaneous current velocity, surface water fluctuation and bottom bed evolution by the use of Boundary Detection Technique (BDT) coupled with PIV technique. In our data sets we successfully captured the migration of ripples, formation of sand bars from the plane sloping bed and interaction of currents and topographic changes.

\section{EXPERIMENTAL SETUPSAND PROCEDURES}

The laboratory experiments were performed in a $30 \mathrm{~m}$ long, $0.6 \mathrm{~m}$ wide, and $0.8 \mathrm{~m}$ deep $2 \mathrm{D}$ wave flume at the University of Tokyo. Progressive waves were generated by piston-type wave maker located at one end of the flume. A schematic diagram of wave flume and apparatus is shown in figure 1. Three HSVCs and two normal video cameras were mounted near the glass wall as shown in figure 1 . HSVCs were used to determine spatial distributions of instantaneous current velocity field on a $1 / 10$ sloping movable sandy bed of uniform sand grains $\left(D_{50}=0.24 \mathrm{~mm}\right)$, while normal video camera were mounted to obtain surface water elevations and bottom bed profile. HSVCs employed in experiments were Casio EX-FH20 that captures images with frame rates of $120 \mathrm{fps}$ and resolutions of $640 \times 480$ pixels. A laser sheet was also introduced from the top to illuminate the suspended sand particles and no special seeding particles were utilized. Laser sheet was set $2 \mathrm{~cm}$ away and parallel to the glass wall to avoid the side wall effects if any. 
The spatial size of field of views (FOVs) by each HSVCs was $190 \times 150 \mathrm{~mm}$, whereas the spatial sizes of normal video camera 1 (outside of the surf zone) and video camera 2 (swash zone) were kept as $430 \times 250 \mathrm{~mm}$ and $230 \times 130 \mathrm{~mm}$ respectively. Spatial bench marks were also marked inside FOVs of each camera to rectify the image results during the analysis. FOVs recorded by $3 \mathrm{HSVCs}$, were later used to determine velocity field distribution over the entire surf zone while surface water elevations and bottom bed profile were computed from images of all five cameras based on BDT.

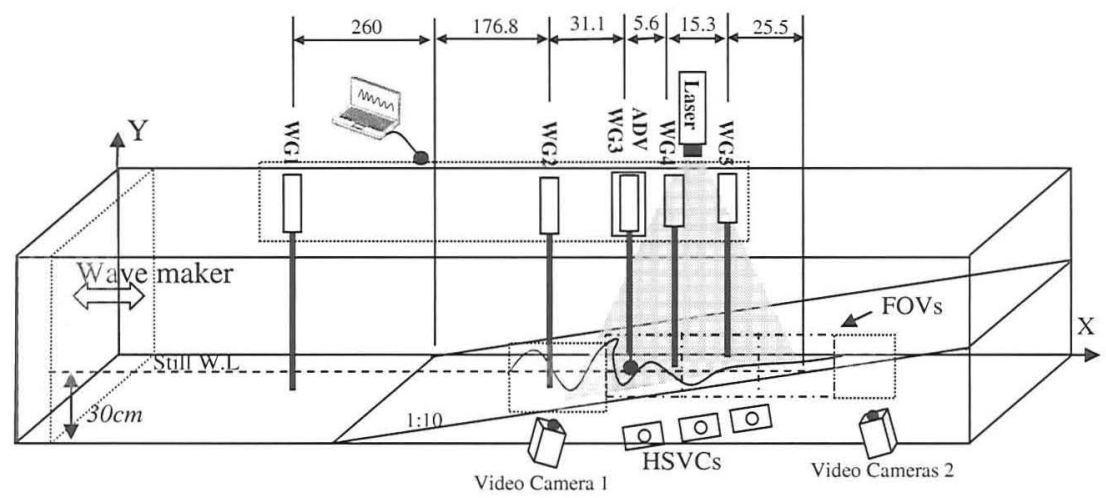

Figure 1: Schematic diagram of wave flume showing apparatus setup and connection details. (All dimensions are in ' $\mathrm{cm}$ ')

Five wave gauges (one at offshore (WG1), one just outside the surf zone (WG2), two inside the surf zone (WG3 \& WG4) and the other one in the swash zone (WG5) were installed for the purpose of validation of BDT. In addition, ADV was also installed outside the breaking point to calibrate the present PIV.

Monochromatic incident waves with heights of $3.31 \mathrm{~cm}$ and period of $1 \mathrm{sec}$ were generated where the water depth was $30 \mathrm{~cm}$. The surf similarity parameter of the incident wave conditions correspond to a plunging type breaker. PIV-based measurements were carried out for about seven minutes and covered 0.57 meters around the breaking point where a bar was formed and moved onshore-ward. To synchronize image data in all these cameras, a high intensity flash was popped up at the end of recordings that was readily detected in each FOV.

\section{DATA ANALYSIS}

\section{Calibration and Validation of PIV}

Since accurate velocity estimation skills using PIV essentially rely on the determinations of window sizes, within which best-fit pixel patterns are searched based on correlations of two successive images, the optimum window size was first calibrated through the comparisons with measured velocities by ADV. Since the installation of ADV probe affected the images for PIV, we performed separate 
experiments respectively for measurements by PIV and ADV after the beach profiles reached to the equilibrium state. In these calibration experiments, both ADV and PIV recorded 20 waves exactly at the same location.

A PIV algorithm, based on enhanced Minimum Quadratic Different (MQD) method (Ahmed \& Sato, 2001), was applied for various combinations of interrogation and searching window sizes. The best match conditions (interrogation window size of $69 \times 69$ pixels and searching window size of 49x49 pixels) were adopted and comparison of results after removal of high frequency noise based on Fast Fourier Transform (fft) are shown in figure 2. Figure 2 compares both horizontal and vertical velocity components measured and estimated by ADV and PIV, respectively.
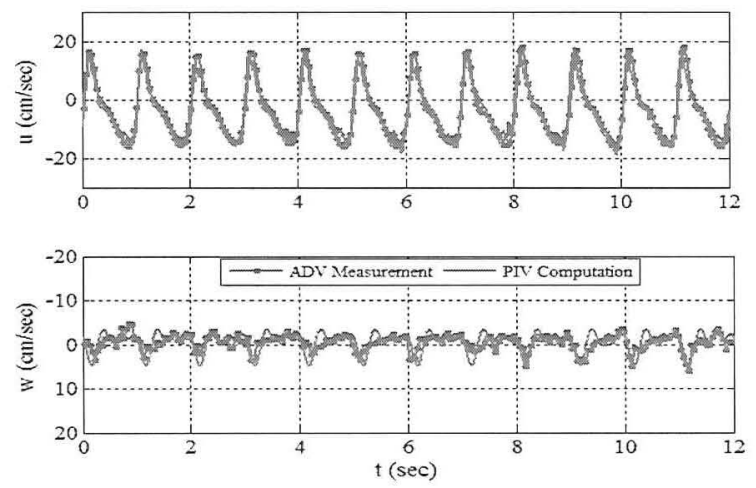

Figure 2: Comparison of PIV computation with ADV measurement for horizontal velocity $u$, and vertical velocity $w$.

The PIV shows excellent agreement for both horizontal and vertical velocities. Relatively high noises found in vertical velocity were due to mismatching of PIV correlation under small velocities of particles and high near bed suspension of sediment. Root-mean-square errors of measured and estimated velocity components in horizontal and vertical directions are 2.6 and $3.2 \mathrm{~cm} / \mathrm{sec}$ respectively.

\section{Boundary Detection Technique}

This study also developed a Boundary Detection Technique (BDT), which captured the temporal and spatial high resolution data of surface water elevations and bottom bed profiles over the entire surf zone. In BDT, RGB information of each image is utilized to obtain threshold values at water-air and water-sand boundaries, respectively. The logic behind the detection of the boundary is to identify the position of pixel where the representative value (say $C$ ) equals the threshold value $\left(\mathrm{C}_{\mathrm{th}}\right)$. The following figure 3(a) shows typical image recorded by HSVC, while 3(b) shows the detected boundaries of surface water and bed bottom in solid lines and the instantaneous velocity fields obtained by PIV in vectors. 

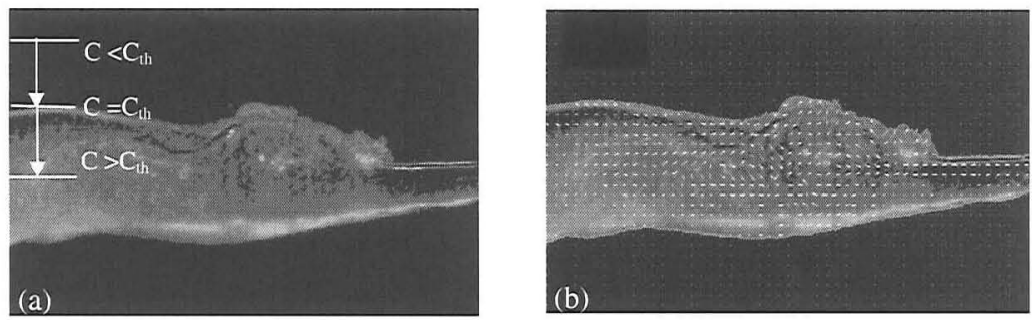

Figure 3: (a).Typical image recorded by HSVC. (b). Water-air and water-sand boundaries detected by BDT (in solid lines) and instantaneous velocity vectors estimated by PIV algorithm.

For validation of BDT, temporal data of surface water elevations from four wave gauges (WG2, WG3, WG4, and WG5) mounted at various positions are compared with BDT results (see figure 4).
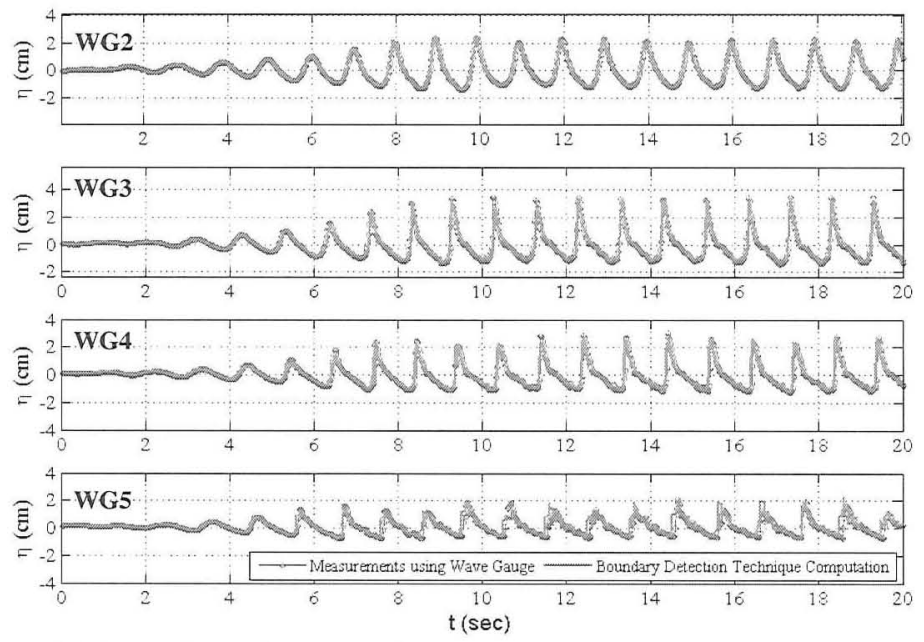

Figure 4: Comparison of water surface elevation, $\eta$ extracted from images using BDT and those recorded by wave gauges at four different locations around the surf zone.

The comparison shows robustness of BDT for measurements of the surface water elevations compared with the one recorded by each wave gauges. Since BDT is sensitive to the local brightness of pixel, therefore air bubbles that develop at the water surface as a result of wave breaking may cause some fluctuations as observed in the comparison of WG5 data. Later, a similar procedure is readily adopted for detection of water-sand boundary and to extracted high resolution bottom bed profiles in temporal and spatial domains. 


\section{DYNAMIC CHANGES OF MEASURED BED PROFILES AND VELOCITIES}

Developed image-capturing techniques were applied for the experimental case respectively at $1,2,3,4,5$ and 6 minutes after the initiation of waves. During the first five minutes, bottom bed profiles and waves were dynamically changed and nearly reached to the equilibrium conditions. At each time (every minute), images of ten waves were used for estimations of detailed velocity components.

\section{Dynamic Changes of Bottom Bed Profiles}

To obtain the bottom bed profile, BDT was applied to the images captured by all the cameras. Based on the known spatial locations at bench marks inside FOVs, obtained boundaries in each image were combined to yield single lines of the bed profiles across the entire surf zone at every time. Figure 5 shows morphology change at $1,2,3,4,5$ and 6 minutes after the initiation of the wave generation.

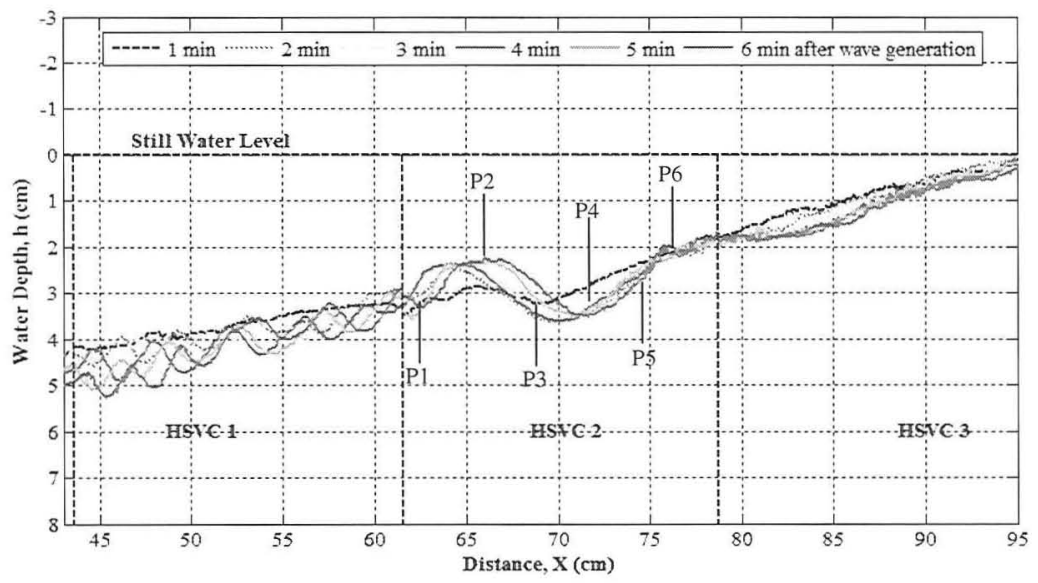

Figure 5: Spatial and temporal variation of bottom bed profile extracted using BDT from the image data recorded with three HSVCs.

As seen in figure 5, BDT successfully captured the bar formation and its onshore migration. It is interesting to observe that the bar was formed quickly within the first two minutes while the onshore movement of the bar was relatively slow but continued until six minutes after the initiation of the wave generation (see region recorded by HSVC 2). Meanwhile, ripples generated outside of the surf zone, seen in HSVC1, show continuous onshore migrations. Further, in the area observed in HSVC3, rapid erosion in near-shore region was observed during the first four minutes. After four minutes, bed profile in the area of HSVC 3 was stabilized while the bar in HSVC 2 still showed onshore migrations. 


\section{Characteristics of Current Velocities and Suspended Sediments over the Bar}

In order to investigate the characteristics of the current and suspended sediment concentrations on the changing movable beds around the bar, six points, we applied PIV at P1, P2, P3, P4, P5 and P6, shown in figure 5, at times, 1 min, when the bar was being developed, $4 \mathrm{~min}$, when the bar was fully developed, and $5 \& 6 \mathrm{~min}$ when the bar was moving shore-ward. The elevation of the observation point was kept constant at 30 pixels $(1 \mathrm{~cm})$ above the movable bed whose elevation changes at different time. At each point, horizontal current velocities, $u$ and image brightness, $C$, which represents the suspended sediment concentrations, were extracted. According to Liu (2005), image brightness and the suspended sediment concentrations have nearly linear relationships under the uniform lighting conditions without the use of additional seeding particles except bottom sand grains for PIV. Figure 6 shows the averaged time profiles of $u$ and $C$ at different time and locations.
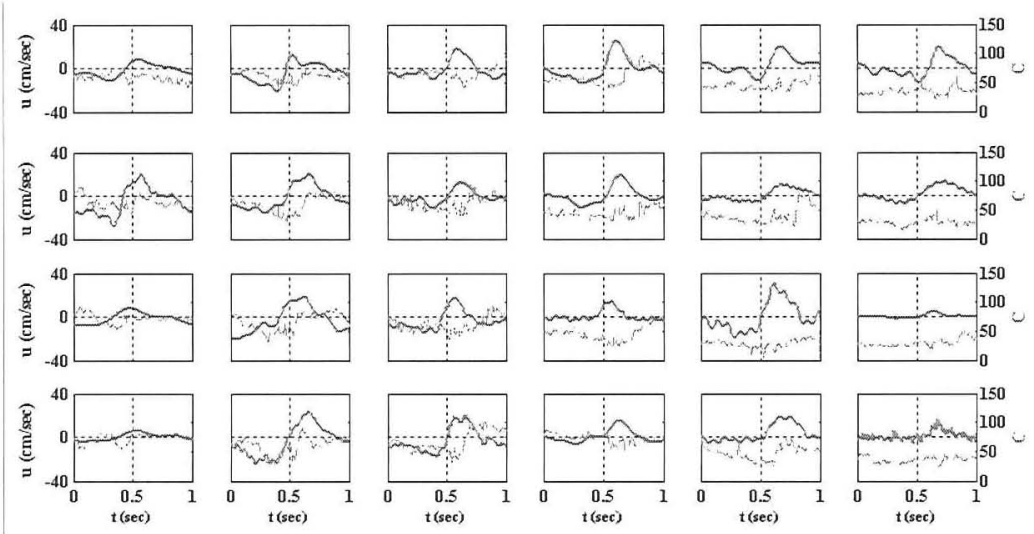

Figure 6: Averaged time profile of horizontal current velocity $u$ (bold lines) and corresponding image brightness, $C$ (dash lines) at P1, P2, P3, P4, P5 and P6 (placed horizontally from left to right) after $1,4,5$ and 6 minutes (placed vertically from top to bottom).

When the absolute values of the horizontal velocity components are relatively small especially seen during the offshore flow, the present PIV appears to yield unreliable velocity estimations due to pixel resolutions of the present setups. However, the points across the sand bar i.e., from P1 to P3, yielded somewhat reliable velocity profiles. Figure 7 shows the estimated mean current velocity and mean suspended sediment concentrations at P1, P2 and P3. 


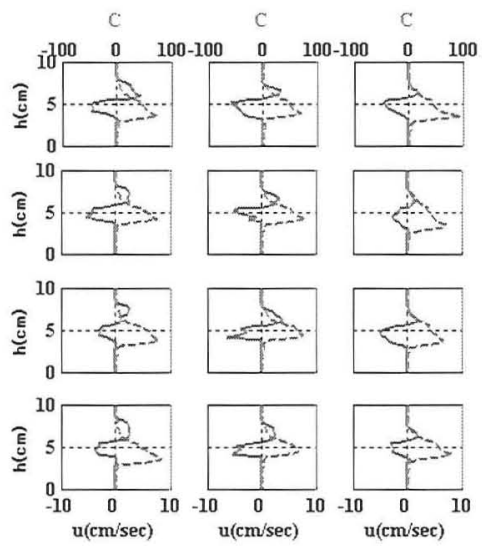

Figure 7: Time averaged undertow velocities (solid line) and suspended sediment concentration (dash line) in terms of brightness at P1, P2 and P3 (placed horizontally from left to right) after 1, 4, 5 and 6 minutes (placed vertically from top to bottom).

\section{Suspended Sediment transport over the Bar}

Net suspended sediment transport rate is quantified by time-averaging the depth-integrated product of $u$ and $C$, i.e.,

$$
q_{s}=\overline{\int_{0}^{h_{t}} u C d z}
$$

with $\mathrm{z}$, upward elevation from the bottom. Note that integration range was limited up to the wave trough level, $h_{t}$, under the assumption that there is nearly no suspended sediments above the trough level. The net $q_{s}$ may then be decomposed into two parts,

$$
\int_{0}^{\overline{h_{t}} u C d z}=\int_{0}^{h_{1}} \bar{u} \bar{C} d z+\int_{0}^{h_{1}} \overline{\tilde{u} \tilde{C}} d z=q_{s 1}+q_{s 2}
$$

with $\bar{u} \& \bar{C}$, the time-averaged components of $u$ and $C$, and $\tilde{u} \& \tilde{C}$, time-fluctuations of $u$ and $C$ around their time-averaged components, i.e., $u=\bar{u}+\tilde{u}$ and $C=\bar{C}+\tilde{C}$.

Figure 8 shows the estimated components of suspended sediment transport rates, $q_{s}, q_{s 1}$ and $q_{s 2}$ at different locations and times. The figure 8 also shows the changing local bottom slopes estimated from the extracted bed profiles. Here the bottom slope is expressed in positive value when the water depth is increasing in the shoreward and the horizontal distance used for estimation of the bottom slope was represented by the excursion amplitude of the bottom orbital velocity.

As seen in the figure $8, q_{s}$, i.e., the depth-integrated product of $\bar{u} \& \bar{C}$, dominate $q_{s 2}$, the time-averaged product of $\tilde{u} \& \tilde{C}$ under all conditions. The net sediment transport, $q_{s}$, at all three points are shoreward and the magnitude of $q_{s}$ at P2 was greater than the others, $\mathrm{P} 1$ and $\mathrm{P} 3$ from $\mathrm{t}=1 \mathrm{~min}$ to $2 \mathrm{~min}$. As a result of this unbalance, bottom bed was eroded between P2 and P3 and accumulated between P1 
and P2. After $\mathrm{t}=3 \mathrm{~min}$, in contrast, net transport at all three locations seems approximately uniform suggesting a temporary equilibrium similar to that observed during morphology changes of bottom bed at corresponding time. At $t=4 \mathrm{~min}$, furthermore, the higher offshore ward transport at P3 relative to P2 caused initiation of shoreward sand bar movement.

The depth-integrated product of mean components, $q_{s l}$, shows strong correlation with the location relative to the bar crest. At P2, for instance, the seaward $q_{s l}$ dramatically increases as the sand bar moves shoreward and the relative location of P2 approached to the bar crest. While $q_{s l}$ shows clear correlation with the relative location to the sand bar, $q_{s 2}$, the component of fluctuating suspended sediment transport rates appear to show correlation with the local bottom slope especially during the first four minutes. Until four minutes, increase or decrease of qs2 surely correspond to the increase or decrease of the bottom slope. It is however interesting to point out that this correlation breaks after five minutes when the movement of the sand bar became predominant.
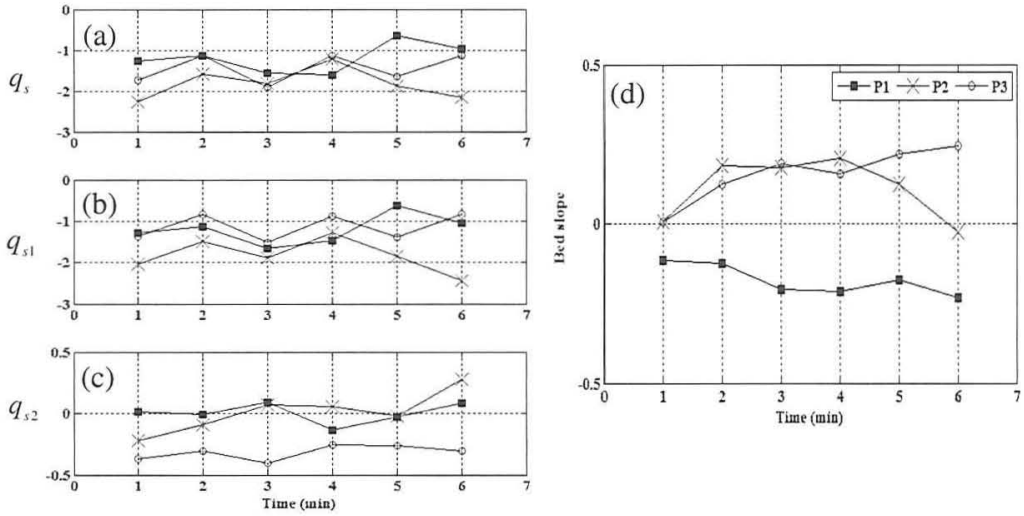

Figure 8: Temporal Variation of $\overline{u C}$ (fig.9a), $\bar{u} \cdot \bar{C}$ (fig. 9b) and $\overline{u^{\prime} C^{\prime}}$ (fig9c) at P1 and P2 and P3. Fig.9d shows the corresponding local bed slope computed across horizontal excursion amplitude of water particle. (Note: A positive value of slope represents downwards slope and vice versa.)

\section{CONCLUSION}

This study developed a new set of image-capturing techniques that successfully obtained the high-resolution quantitative data sets of waves, currents and bottom bed profiles both in the time and space domains. Boundary Detection Technique successfully captured the phenomenon of bar formation and its onshore migration along with ripple migrations.

Change of the time-profiles of current velocity as well as suspended sediment concentrations were estimated and, based on these profiles, the time-averaged 
components of net suspended sediment transport rates were computed and compared. The total net suspended sediment transport rates reasonably explained the morphology change around the sand bar. The net suspended sediment transport rates were decomposed into two parts, mean-component, $q_{s I}$, and the fluctuation components, $q_{s 2}$. The mean components dominantly determined the total net suspended sediment transport rates and their magnitudes show clear correlations with the relative locations to the sand bar crest. While the magnitude was relatively small, the fluctuating component showed clear correlations with the local bottom slopes especially before the shoreward movement of the developed sand bar. Further improvements of the image-capturing system and investigations of obtained highresolution data sets of current, suspended sediments and corresponding bottom topography should be achieved in the future research.

\section{ACKNOWLEDGMENT}

This work was gratefully supported by Grant-in-Aid for Young Scientists (B), No. 20760322, from Japan Society for the Promotion of Science (JSPS).

\section{REFERENCES}

Ahmed, A. S. M., and Sato S. (2001). "Investigation of bottom boundary layer dynamics of movable bed by using enhanced PIV technique" Coastal Engineering Journal, Vol. 43, No. 4, pp. 239258.

Cox, D.T., and Kobayashi, N., (2000). "Identification of intense, intermittent coherent motions under shoaling and breaking waves". J. Geophysical Research. 105 (6), 223-236.

Cowen, E. A., Sou, I. M., Liu, L. F, and Raubenheimer, B. (2003). "Particle image velocimetry measurements within a laboratory-generated swash zone". Journal of Engineering Mechanics 1119-1129.

Elgar, S., Rauhenheimer, and B., Guza, R.T. (2005). "Quality control of acoustic doppler velocimeter data in the surf zone". Meas. Sci. Technol. 16, 1889-1893.

Govender, K., Mocke, G.P., and Alport, M.J. (2002). "Video-imaged surf zone wave and roller structures and flow fields" J. of Geophysical Research, Vol. 107, No. C7, 3072.

Govender, K., Mocke, G.P., and Alport, M.J. (2004). "Dissipation of isotropic turbulence and length scale measurements through the wave roller in the laboratory spilling waves" $J$. of Geophysical Research, Vol, 109 C08018.

Govender, K., Michallet, H., Alport, M.J., Pillay, U., Mocke, G.P., and Mory, M. (2009). "Video DCIV measurements of mass and momentum fluxes and kinetic energies in laboratory waves breaking over a bar". Coastal Engineering (56) 876-885.

Liu, H., (2005). "Laboratory experiments and numerical simulation on sheetflow sediment movement" $P h D$ Thesis, Dept. of Civil Engineering, The University of Tokyo.

Longo, S., (2009). "Vorticity and intermittency within the pre-breaking region of spilling breakers." Coastal. Engineering. 56 (3), 285-296.

Nadaoka, K., Hino, M., and Koyano, Y., (1989). "Structure of the turbulent flow field under breaking waves in the surf zone". J. Fluid Mech. 204, 359-387.

Kimmoun O., and Branger, H. (2007). "A particle image velocimetry investigation on laboratory surfzone breaking waves over a sloping beach" J. Fluid Mechanics., vol.588, 353-397.

Shin, S. and Cox. D.T., (2006). "Laboratory observations of inner surf and swash-zone hydrodynamics on a steep slope. Continental Shelf Research 26. 561-573.

Stive, M.J.F. (1984). "Energy dissipation in wave breaking on gentle slopes" Coastal Engineering 8, 99-127.

Ting, F.C.K., and Kirby, J.T., (1996). "Dynamics of surf-zone turbulence in a spilling breaker". Coastal. Engineering. 27, 131-160.

Huang, Z. C., Hsiao S. C., Hwung H. H., and Chang, K. A. (2009). "Turbulence and energy dissipation of surf-zoen spilling breakers", Coastal Engineering (56) 733-746. 